\title{
Acoustic Tomography for Scalar and Vector Fields: Theory and Application to Temperature and Wind Estimation
}

\author{
IVANa Jovanović, Luciano Sbaiz, AND Martin VeTTERLi \\ Audiovisual Communications Laboratory, Ecole Polytechnique Fédérale de Lausanne, \\ Lausanne, Switzerland
}

(Manuscript received 9 December 2008, in final form 2 March 2009)

\begin{abstract}
Acoustic tomography is a type of inverse problem. The idea of estimating physical quantities that influence sound propagation by measuring the parameters of propagation has proven to be successful in many practical domains, including temperature and wind estimation in the atmosphere. However, in most of the previous work in this area, the algorithms used have not been proven mathematically to provide the correct solution to the inverse problem.

This paper considers the problem of reconstructing 2D temperature and wind fields by using acoustic tomography setups. Primarily, it shows that the classical time-of-flight measurements are not sufficient to reconstruct wind fields. As a solution, an additional set of measurements related solely to the parameters of sound propagation-more precisely, to the angles of departure/arrival of sound waves-is suggested. To take the full benefit of this additional information, the bent-ray model of sound propagation is introduced. In this work, it is also shown that, when a temperature and a source-free $2 \mathrm{D}$ wind field are observed on bounded domains, the complete reconstruction is possible using only measurements of the time of flight. Conversely, the angles of departures/arrivals are sufficient to reconstruct a temperature and a curl-free 2D wind fields on bounded domains. Further, an iterative reconstruction algorithm is proposed and possible variations to the main scheme are discussed. Finally, the performed numerical simulations confirm the theoretical results, demonstrate fast convergence, and show the advantages of the adopted bent-ray model for sound propagation over the straight-ray model.
\end{abstract}

\section{Introduction}

Tomography aims at recovering an unknown multidimensional field based on the interactions between the considered medium and the signals that are emitted by radiating devices and captured by appropriate sensors. For many decades, tomography methods have been widely used in physics, geophysics, medicine, and technology for nondestructive testing. Examples include the use of magnetic resonance imaging to detect physiological alterations of living tissues (Haacke et al. 1999) and seismic tomography to image the interior of the earth (Stewart 1991). The success of the tomographic approach primarily stems from its noninvasive nature and the fact that a significantly larger amount of data can

Corresponding author address: Ivana Jovanović, Audiovisual Communications Laboratory, BC320 Station 14, Ecole Polytechnique Fédérale de Lausanne (EPFL), 1015 Lausanne, Switzerland.

E-mail: ivana.jovanovic@epfl.ch be obtained compared to the classical one-sensor-onemeasurement setup. Furthermore, tomographic measurements acquire a global, as opposed to punctual, knowledge of the measured field.

Acoustic tomography for monitoring phenomena in the atmosphere, particulary temperature and wind, was first proposed in the 1990s (Spiesberger and Fristrup 1990; Wilson and Thomson 1994) as an attempt to use the techniques successfully applied in monitoring ocean structure (Munk and Wunsch 1982). The application of acoustic tomography is enabled by the strong dependence of sound propagation on the spatial distribution of air temperature and wind flow. Moreover, imaging these physical quantities in near-ground atmosphere with acoustic tomography has been already demonstrated in field experiments (Wilson and Thomson 1994; Chunchuzov et al. 1997; Ziemann et al. 1999b). However, in most of the previous work, the used algorithms have not been proven mathematically to provide the correct solution to the inverse problem. Hence, the goal of this paper is to bring forth related material from 
adjacent fields of research and to present some known, as well as new, results. In particular, the questions of joint two-dimensional (2D) temperature and wind field reconstruction, for general and specific cases of wind field, are settled.

\section{a. Temperature and wind field estimation}

The temperature estimation is known to be a scalar tomography problem in the sense that it amounts recovering a scalar function from its line integrals by means of the Radon transform and its inverse. ${ }^{1}$ These integrals can be typically computed from the time taken by a sound wave to propagate from a transmitter to a receiver, referred to as "time of flight." The reconstruction of wind fields, however, deals with vector tomography, in which the unknown field is described by a vector function. Although time of flight is theoretically sufficient to recover the complete wind field, the inverse problem that needs to be solved in this case is highly nonlinear, hence analytically intractable. Thus, it is then necessary to resort to linearization, which, although attractive from a computational standpoint, seriously limits the reconstruction ability. In fact, Johnson et al. (1977) were the first to notice that linearizing the relationship between the time of flight and the wind field makes that one wind component become "invisible" to the reconstruction process. Later, Norton (1992) laid the groundwork for a theoretical treatment of this problem by showing that, according to Helmholtz's theorem, every vector field can be written as the sum of an irrotational (or curl free) and a solenoidal (or source free) component and that only the solenoidal part can be imaged from the time of flight. Additional measurements are thus required to reconstruct the missing component. It should be noted that this essential phenomenon is often left out in the literature of acoustic tomography in the atmosphere. This has an unpredictable effect on the reconstruction and perpetuates the incorrect belief that the inverse problem is illconditioned, whereas it is actually ill-posed.

In this paper, an additional set of acoustic measurements, related to the angles of departure and arrival of sound waves, is suggested. An iterative algorithm that uses both the time-of-flight and the angle-of-departure/ arrival measurements to recover the full temperature and wind field is proposed. Note that a related approach was suggested by Braun and Hauck (1991) in the con-

\footnotetext{
${ }^{1}$ Strictly speaking, because the sound rays bend when propagating through inhomogeneous medium, the generalized Radon transform needs to be applied.
}

text of fluid tomography. They proposed an additional set of measurements based on an optical Schlieren technique. However, their method is only applicable in rather specialized scenarios with an optical access, whereas our method is solely based on sound propagation.

\section{b. Bounded domains and special cases of wind fields}

In most physical setups, we observe an unknown field on a bounded domain where the boundary corresponds to the closed curve on which the transmitters and receivers reside. The reconstruction of source-free vector fields observed on a bounded domain is an important problem and is studied in detail in the present manuscript. Because the bounded domain admits harmonic vector fields that are both irrotational and solenoidal, the Helmholtz decomposition of the field is not any more unique. The commonly studied transform-based solutions (e.g., the solutions given by the Radon and Fourier transforms) fail to give the correct result because they separately reconstruct the solenoidal and irrotational components. In this case, it is no longer clear which part of the solenoidal component is reconstructed. To overcome this problem, Norton (1992) proposed to measure the normal component of the source-free wind (vector) field on the boundary in addition to the time of flight.

Instead, we show that the time of flight is sufficient for the reconstruction of a source-free wind field on a bounded domain; that is, no additional measurements are needed. Moreover, the estimation of the wind field is reduced to the estimation of its solenoidal component and, consequently, the dimension of the problem is reduced to half of the original one. We also design an algorithm that reconstructs the temperature and the source-free wind field from the time-of-flight measurements.

Other special cases of wind fields, namely, (i) the 2D wind fields that are obtained as a slice of a 3D sourcefree wind and (ii) irrotational wind fields, are also studied. It is shown what types of measurements are needed for their reconstruction.

\section{c. Outline and notations}

This paper is organized as follows: section 2 gives a brief introduction to inverse problems, presents the theory of sound propagation, introduces the bent-ray model, and shows the limitations of the time-of-flight measurements. It then emphasizes the importance of Helmholtz's theorem and introduces the angle-of-departure/arrival measurements. A new method to obtain these measurements is also proposed. In section 3, important practical scenarios are discussed, emphasizing the cases for which the required measurements can be reduced to only one of 
the two proposed sets. Section 4 describes the algorithms needed for reconstruction of both general and special temperature and wind field cases. The simulation results are shown in section 5. Finally, in section 6, we give concluding remarks.

Vectors and matrices will be noted in bold and the mean (or reference) value of any parameter $f$ will be noted as $f_{0}$. The scalar product of two vectors will be denoted with . and the vector product will be denoted with $\times$.

\section{The inverse problem in acoustic tomography}

\section{a. General formulation}

In the usual terminology of inverse problems (Wilson and Thomson 1994), the set of parameters to be determined, which describes the state of the field, is called the model. We denote the model as $\mathcal{M}$. To obtain the information on the model parameters, measurements of some observable parameters are needed. The experimental measurements are called the data $\mathcal{D}$. To compute the model parameters, first the forward problem is defined by devising a mapping $\mathcal{G}$ such that $\mathcal{D}=\mathcal{G} \mathcal{M}$. The inverse mapping $\mathcal{M}=\mathcal{G}^{-1} \mathcal{D}$ is then constructed from the forward mapping.

Usually solving a nonlinear problem is analytically not possible and computationally intractable; thus, forward problems are often linearized. Commonly, it is done by using the Taylor series expansion in terms of the models,

$$
\mathcal{D}=\mathcal{G M}_{0}+\mathcal{G}_{l}\left(\mathcal{M}-\mathcal{M}_{0}\right)+\cdots,
$$

and keeping only the first-order terms, so that

$$
\mathcal{D}-\mathcal{D}_{0}=\mathcal{G}_{l}\left(\mathcal{M}-\mathcal{M}_{0}\right),
$$

where $\mathcal{G}_{l}$ is a linearized mapping around the unperturbated model $\mathcal{M}_{0}$. Although inverse problems are often formulated in infinite dimensional spaces (or continuous domain), limitations to a finite number of measurements and the practical consideration of recovering only a finite number of unknown parameters usually lead to the problems being recast in discrete form. The discrete version of the problem is going to be discussed in the reconstruction algorithms; however, for showing the existence and the uniqueness of solution, we will use the more convenient continuous form.

In the rest of this section, our goal is to define a set of data acquired by acoustic tomography that enables wind and temperature reconstruction. The choice, of course, depends on the physics of sound propagation.

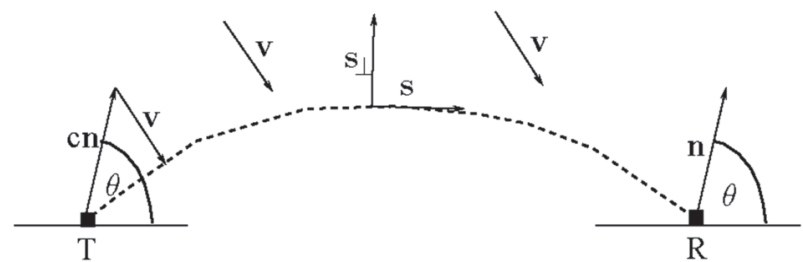

FIG. 1. Sound propagation. An example of a ray trajectory with the following notation: $\mathrm{T}$ is the transmitter; $\mathrm{R}$ is the receiver; $c$ is the sound speed; $\mathbf{n}=[\cos \theta \sin \theta]$ is the unit vector normal to the wave front; $\mathbf{v}$ is the wind field; $\mathbf{s}$ is the unit vector tangent to the trajectory; and $\mathbf{s}_{\perp}$ is the unit vector orthogonal to $\mathbf{s}$.

\section{b. Sound propagation in the atmosphere and the influence of wind and temperature}

The propagation of sound waves in an inhomogeneous moving medium, e.g., the atmosphere, is completely determined by the system of linearized equations of fluid dynamics. When the medium inhomogeneities are large compared to the wavelength, the energy propagation is well described by the ray theory of sound propagation. The most simplified ray model is the straight-ray model, and it has been widely used in the previous research (Ziemann et al. 1999a; Barth et al. 2006). In this study, a more accurate model is adopted (the bent-ray model) that accounts for ray refraction.

The sound propagation is described by the group velocity and a vector $\mathbf{b}$, which is defined below (Ostashev 1997). The group velocity $\mathbf{u}_{g}$ is given by

$$
\frac{d \mathbf{x}}{d t}=\mathbf{u}_{g}=c \mathbf{n}+\mathbf{v}
$$

where $\mathbf{x}$ is the position, $t$ is the time, $c$ is the sound speed, $\mathbf{n}$ is the unit vector normal to the wave front, and $\mathbf{v}$ is the wind velocity (see Fig. 1). The vector $\mathbf{b}$ is defined as $\mathbf{b}=\mathbf{k} / k_{0}$, where $\mathbf{k}$ being the wave vector. The direction of $\mathbf{b}$ is normal to the wave front, and $\mathbf{b}$ changes in time as

$$
\frac{d \mathbf{b}}{d t}=-\frac{c_{0} \nabla c}{c}-\nabla(\mathbf{b} \cdot \mathbf{v})+\frac{(\mathbf{b} \cdot \mathbf{v}) \nabla c}{c},
$$

where $\nabla$ is the gradient operator. From Eqs. (2) and (3), the ray path can be computed by imposing some initial conditions on $\mathbf{x}$ and $\mathbf{b}$. For example, the starting point may correspond to the transmitter position $\mathbf{x}(0)=\mathbf{x}_{T}$, and the initial $\mathbf{b}(0)$ is chosen such that the ray reaches the receiver while ensuring that at every point, $k+\mathbf{k} \cdot \mathbf{v} / c=$ $k_{0} c_{0} / c$. Also, the sound speed depends on the temperature as

$$
c=20.05 \sqrt{T(1+0.511 q)},
$$


where $T$ is the air temperature measured in kelvins, the value 20.05 stands for the square root of the product of specific gas constant for dry air $\left(287.05 \mathrm{~J} \mathrm{~kg}^{-1} \mathrm{~K}^{-1}\right)$ and the ratio of specific heat at constant volume and constant pressure for dry air (1.4), and $q$ is the specific humidity of the air (usually between 0 and $0.03 \mathrm{~kg} \mathrm{~kg}^{-1}$ ). Finally, taking into account Eqs. (2)-(4), it can be concluded that the sound propagation in the atmosphere strongly depends on temperature distribution and wind flow. In the following, we are going to see the observable parameters of the sound propagation that will permit the reconstruction of the temperature and wind fields. Because the temperature is uniquely determined from the sound speed, the aim will be to reconstruct $c$ and $\mathbf{v}$.

\section{c. Time-of-flight measurements and linearization}

Time-of-flight measurements are the classical measurements provided by acoustic tomography methods. The time of flight from a transmitter to a receiver is equal to

$$
\tau=\int_{\Gamma} \frac{1}{(c \mathbf{n}+\mathbf{v}) \cdot \mathbf{s}} d s
$$

where $\mathbf{s}$ is the unit vector tangent to the ray path $\Gamma$ and parallel to the group velocity. Note that time of flight contains the information on $c$ and $\mathbf{v}$, but it is rather impractical to choose Eq. (5) as the forward model. Instead, Eq. (5) is linearized as

$$
\begin{aligned}
\tau & \simeq \int_{\Gamma} \frac{1}{c_{0} \mathbf{n} \cdot \mathbf{s}} d s-\int_{\Gamma} \frac{(\Delta c \mathbf{n}+\mathbf{v}) \cdot \mathbf{s}}{\left(c_{0} \mathbf{n} \cdot \mathbf{s}\right)^{2}} d s \\
& \simeq \tau_{0}-\frac{1}{c_{0}^{2}} \int_{\Gamma}(\Delta c \mathbf{n}+\mathbf{v}) \cdot d \mathbf{s}
\end{aligned}
$$

where $\Delta c=c-c_{0}$ and $\Delta c$ and $\|\mathbf{v}\|$ are typically much smaller than $c_{0}$. Now, the time-of-flight perturbations are linearly related to $\Delta c$ and $\mathbf{v}$, as

$$
\left(\tau_{0}-\tau\right) c_{0}^{2}=\int_{\Gamma}(\Delta c \mathbf{n}+\mathbf{v}) \cdot d \mathbf{s} .
$$

The line integral on the right-hand side of Eq. (6) is also called the longitudinal interaction $l_{\Gamma}$ by analogy with the line integrals of the vector field defined in Braun and Hauck (1991), namely

$$
l_{\Gamma}=\int_{\Gamma}(\Delta c \mathbf{n}+\mathbf{v}) \cdot d \mathbf{s}
$$

It is important to check if we can uniquely determine the changes in $c$ and $\mathbf{v}$ from Eq. (7) or if $l_{\Gamma}$ is sufficient for estimating $c$ and $\mathbf{v}$. The following example shows that it is not the case.

\section{EXAMPLE 1 (INVISIBLE WIND)}

Assume the synthetic wind field shown in Fig. 2 [i.e., $\left.\mathbf{v}_{1}(\mathbf{r})=\mathbf{r}\right]$ and a particular measurement setup (i.e., the transmitters and the receivers are placed on a circle centered at the origin). For simplicity of exposure, the temperature is assumed to be constant $(\Delta c=0)$. Using the previously proposed linearization, the time of flight between any two points on the boundary $\partial D$ is

$$
\tau \simeq \int_{-L}^{L} \frac{1}{c_{0}} d s-\frac{1}{c_{0}^{2}} \int_{-L}^{L} \mathbf{v}_{1} \cdot d \mathbf{s}=\frac{2 L}{c_{0}}=\tau_{0}
$$

because the integral of an odd function over a symmetric interval is always equal to zero. As a result, the first-order time-of-flight perturbations or, equivalently, the longitudinal interactions do not depend on this particular wind and hence no information on the field can be obtained whatsoever. More importantly, in our model, the time-of-flight perturbations $\tau_{2}$ caused by any other "realistic" wind field $\mathbf{v}_{2}$ would not differ from the perturbations caused by the wind $\mathbf{v}_{2}+\mathbf{v}_{1}$, because $\mathbf{v}_{1}$ shows to be an "invisible wind." Conversely, if we measure the time of flight $\tau_{2}$, what is the underlying wind? Now, it is clear that both $\mathbf{v}_{2}$ and $\mathbf{v}_{2}+\mathbf{v}_{1}$ are the correct answers and the inverse problem has no unique solution. Therefore, we need more information to resolve this ambiguity. Again, the nonlinear model provides more information because

$$
\begin{aligned}
\tau & =\int_{-L}^{L} \frac{1}{\left(c_{0} \mathbf{n}+\mathbf{v}_{1}\right) \cdot \mathbf{s}} d s=\int_{-L}^{L} \frac{1}{c_{0} \mathbf{n} \cdot \mathbf{s}+\mathbf{r} \cdot \mathbf{s}} d s, \\
& =\int_{-L}^{L} \frac{1}{c_{0}+s} d s=\log \left(c_{0}+L\right)-\log \left(c_{0}-L\right) \neq \tau_{0},
\end{aligned}
$$

but it is of little practical interest, because of the difficulty in solving a nonlinear inverse problem.

\section{d. Vector tomography}

Vector function cannot be recovered from the values of its line integrals. The latter become obvious if one considers the Helmholtz decomposition of vector fields. Recall that according to Helmholtz's theorem, every vector field can be decomposed into an irrotational $\mathbf{v}_{I}$ (or curl-free $\nabla \times \mathbf{v}_{I}=0$ ) and solenoidal $\mathbf{v}_{S}$ (or sourcefree $\boldsymbol{\nabla} \cdot \mathbf{v}_{S}=0$ ) component

$$
\mathbf{v}(\mathbf{x})=\mathbf{v}_{I}(\mathbf{x})+\mathbf{v}_{S}(\mathbf{x}) .
$$




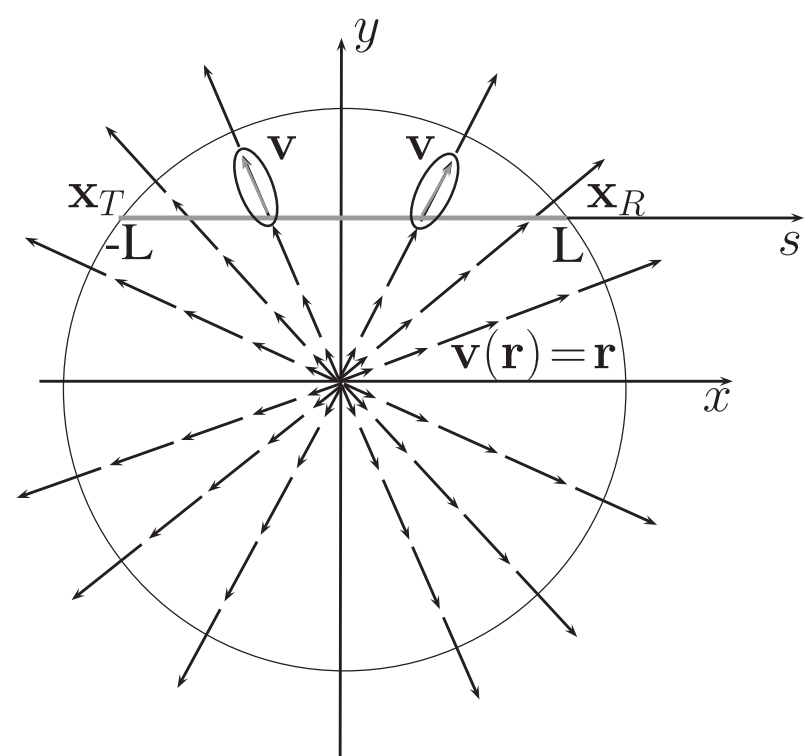

FIG. 2. One example of an invisible wind field. The wind field $\mathbf{v}$ is assumed to be radial: $\mathbf{v}(\mathbf{r})=\mathbf{r}$. For any two points on the boundary $\mathbf{x}_{T}$ and $\mathbf{x}_{R}$, the longitudinal interactions (i.e., the line integral of the vector field) sum up to zero.

The decomposition is not unique because there exist fields (referred to as harmonic fields) that can be both curl-free and source-free. For example, in a bounded domain $D$, the harmonic fields are the special cases of curl-free fields having the sources outside the domain $D$ or the source-free fields whose curls are closing outside the domain $D$. Sometimes, it is appropriate to separate this third harmonic component and to make the decomposition unique, but for now we will keep the two-component representation. The two components can be described using potential functions

$$
\begin{aligned}
\mathbf{v}_{I}(\mathbf{x}) & =\nabla \phi(\mathbf{x}), \\
\mathbf{v}_{S}(\mathbf{x}) & =\nabla \times \boldsymbol{\psi}(\mathbf{x}),
\end{aligned}
$$

where $\phi$ and $\boldsymbol{\psi}$ are the scalar and vectorial potentials of the field $\mathbf{v}$, respectively. For a 2D field (e.g., in the $x-y$ plane), $\boldsymbol{\psi}$ has only a component along the $z$ axis: $\boldsymbol{\psi}=\psi \mathbf{e}_{z}$. The representation using potentials is equivalent to the full knowledge of the vector field and it amounts to representing a vector field with the two scalar functions $\phi(\mathbf{x})$ and $\psi(\mathbf{x})$. Applying the Helmholtz decomposition on $\mathbf{v}$ in Eq. (7) and assuming, for simplicity, that $\Delta c=0$, we have

$$
\begin{aligned}
l_{\Gamma} & =\int_{\Gamma}(\nabla \phi+\nabla \times \boldsymbol{\psi}) \cdot d \mathbf{s}, \\
& =\phi\left(\mathbf{x}_{R}\right)-\phi\left(\mathbf{x}_{T}\right)+\int_{\Gamma} \frac{\partial \psi}{\partial y} s_{x}-\frac{\partial \psi}{\partial x} s_{y} d s,
\end{aligned}
$$

where the latter equality comes from the gradient theorem; that is, $\int_{a}^{b} \boldsymbol{\nabla} f \cdot d \mathbf{s}=f(b)-f(a)$. Notice that, except for the boundary values $\phi\left(\mathbf{x}_{R}\right)$ and $\phi\left(\mathbf{x}_{T}\right)$, the longitudinal interaction does not give any information on the irrotational wind component.

\section{e. Additional set of measurements for irrotational wind component}

In the past, several researchers working in the field of fluid tomography came to the conclusion that time-of-flight measurements are not sufficient for vector field reconstruction. Braun and Hauck (1991) proposed a new set of measurements, called the transversal interactions, which corresponds to the integration of the normal component of the vector field along the propagation path

$$
t_{\Gamma}=\int_{\Gamma} \mathbf{v} \cdot d \mathbf{s}_{\perp}
$$

where $\mathbf{s}_{\perp}$ is normal to $\mathbf{s}$, as shown in Fig. 1. The transversal interaction provides the information on the irrotational component of the vector field because

$$
\begin{aligned}
t_{\Gamma} & =\int_{\Gamma}(\nabla \phi+\nabla \times \psi) \cdot d \mathbf{s}_{\perp} \\
& =-\psi\left(\mathbf{x}_{R}\right)+\psi\left(\mathbf{x}_{T}\right)+\int_{\Gamma} \frac{\partial \phi}{\partial y} s_{x}-\frac{\partial \phi}{\partial x} s_{y} d s .
\end{aligned}
$$

To obtain the transversal interaction, we suggest a new method based solely on acoustic measurements.

Let us first slightly modify the definition of the transversal interaction so that it also takes into account the temperature fields; that is,

$$
t_{\Gamma}=\int_{\Gamma}(\Delta c \mathbf{n}+\mathbf{v}) \cdot d \mathbf{s}_{\perp} .
$$

Notice that the group velocity along the trajectory is tangent to the trajectory; hence, at every point,

$$
\left(c_{0} \mathbf{n}+\Delta c \mathbf{n}+\mathbf{v}\right) \cdot \mathbf{s}_{\perp}=0 .
$$

The transversal interaction can thus be written as

$$
t_{\Gamma}=-c_{0} \int_{\Gamma} \mathbf{n} \cdot d \mathbf{s}_{\perp} \cdot
$$

To estimate $t_{\Gamma}$, we need to know $\mathbf{n}=(\cos \theta \sin \theta)^{T}$ along the trajectory $\Gamma$. As a first approximation, it can be supposed that $\mathbf{n}$ is constant along $\Gamma$, which is true when the temperature and wind fields are uniform (Sbaiz and Vetterli 2003). Then, 

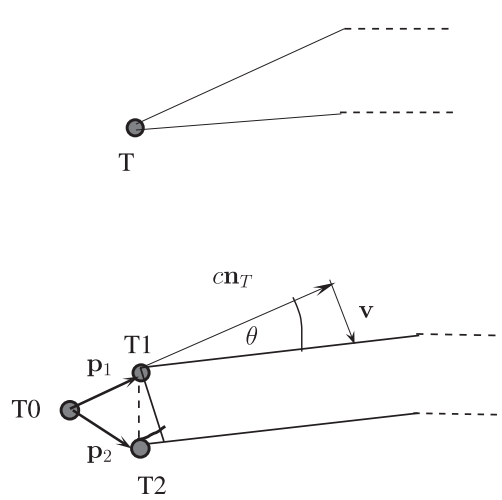

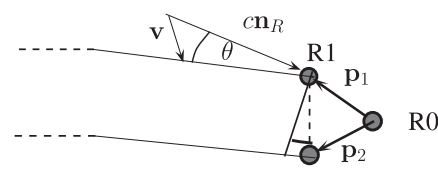

R2

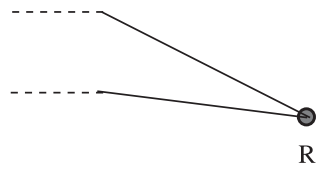

FIG. 3. The vectors $\mathbf{n}_{T}$ and $\mathbf{n}_{R}$ can be estimated by measuring the angles of departure and arrival of the sound wave, and by measuring $\mathbf{v}$ and $\mathbf{u}_{g}$ locally at the transmitter $\mathrm{T}$ or the receiver $\mathrm{R}$ side. The angles of departure and arrival and the local wind measurements are measured using the acoustic tripole (i.e., T0-T2 and R0-R2).

$$
\begin{aligned}
t_{\Gamma} & =c_{0} \int_{\Gamma}\left(\begin{array}{c}
\cos \theta \\
\sin \theta
\end{array}\right) \cdot\left(\mathbf{s} \times \mathbf{e}_{z}\right) d s \\
& \simeq c_{0}\left[\cos \theta\left(y_{R}-y_{T}\right)-\sin \theta\left(x_{R}-x_{T}\right)\right],
\end{aligned}
$$

where $\mathbf{s}_{\perp}=-\mathbf{s} \times \mathbf{e}_{z}$, and $t_{\Gamma}$ can be approximately computed if we know the angle $\theta$ and the exact position of the transmitter and the receiver. A better approximation of $t_{\Gamma}$ can be obtained if we take $\theta=\left(\theta_{T}+\theta_{R}\right) / 2$, where $\theta_{T}$ and $\theta_{R}$ are the corresponding angles at the transmitter and the receiver side, respectively. Possible further improvement can be achieved if we assume that $\Gamma$ is known (from the previous iteration) and that the vector $\mathbf{n}$ changes linearly from $\mathbf{n}_{T}$ at the transmitter side to $\mathbf{n}_{R}$ at the receiver side. It is also important to add that the approximation in Eq. (8) will only be applied to the error fields, and only the difference between $\mathbf{n}$ and its current estimate $\hat{\mathbf{n}}$ is assumed to be constant. The error introduced by the approximation will be evaluated in the numerical example 1 of section 5 .

The angles $\theta_{T}$ and $\theta_{R}$ can be estimated from the angles of departure/arrival of the sound wave, as explained in the following. Assume we want to measure the angle $\theta_{R}$. This angle can be estimated from $\mathbf{n}_{R}$, which is found as

$$
\mathbf{n}_{R}=\frac{\mathbf{u}_{g}-\mathbf{v}}{\left\|\mathbf{u}_{g}-\mathbf{v}\right\|} .
$$

The group velocity $\mathbf{u}_{g}$ can be found using the acoustic tripole shown in Fig. 3. We measure the time of flight for R0, R1, and R2 and set $\Delta \tau_{1}=\tau_{\mathrm{R} 1}-\tau_{\mathrm{R} 0}$ and $\Delta \tau_{2}=$ $\tau_{\mathrm{R} 2}-\tau_{\mathrm{R} 0}$, where $\tau_{\mathrm{R} i}$ is the time of flight measured at the receiver $\mathrm{R}_{i}$. Then, it holds that

$$
\mathbf{u}_{g} \cdot \mathbf{p}_{1}=\left\|\mathbf{u}_{g}\right\|^{2} \Delta \tau_{1} \quad \text { and }
$$

$$
\mathbf{u}_{g} \cdot \mathbf{p}_{2}=\left\|\mathbf{u}_{g}\right\|^{2} \Delta \tau_{2}
$$

where $\mathbf{p}_{1}$ and $\mathbf{p}_{1}$ are shown in Fig. 3. The previous two equations are both the acoustic dipole equation. We need two of them because the speed $\mathbf{u}_{g}$ is unknown. From Eqs. (10) and (11), we can find $\mathbf{u}_{g}$. The same configuration of the acoustic tripole can be used to measure the local wind $\mathbf{v}$ at the receiver side. This can be done using the principles of the sonic anemometer (Coppin and Taylor 1983). Once $\mathbf{u}_{g}$ and $\mathbf{v}$ are estimated, we can compute $\mathbf{n}_{R}$ and the angle $\theta_{R}$ from Eq. (9). The method dual to this one can be used to measure the angle $\theta_{T}$. In this case, we would use the time of flight from the transmitters $\mathrm{T} 0, \mathrm{~T} 1$, and $\mathrm{T} 2$ (see Fig. 3).

\section{f. Definition of the inverse problem}

Finally, the transversal and the longitudinal interactions together uniquely determine the temperature and the wind field and they both can be estimated by measuring the parameters of sound propagation. The correct general formulation of the forward problem is then

$$
\begin{aligned}
& l_{\Gamma}=\int_{\Gamma}(\Delta c \mathbf{n}+\mathbf{v}) \cdot d \mathbf{s} \quad \text { and } \\
& t_{\Gamma}=\int_{\Gamma}(\Delta c \mathbf{n}+\mathbf{v}) \cdot d \mathbf{s}_{\perp} .
\end{aligned}
$$

In the following, important practical scenarios will be discussed emphasizing the situations in which the set of measurements can be reduced to either Eq. (12a) or Eq. (12b). Before we continue, note that when $\mathbf{v}=0$, the temperature estimation can be obtained from either Eq. (12a) or Eq. (12b). In this case, the problem reduces to a scalar tomography problem. 


\section{Important practical cases}

There are important practical scenarios that deserve to be studied in more detail. They are all related to the special cases of wind field because the temperature estimation was shown to be an "easy" problem. Also, they all consider the physical fields on a bounded region.

\section{a. $2 D$ projection of a $3 D$ source-free wind field}

In a usual practical scenario, the $2 \mathrm{D}$ wind field that will be reconstructed represents a slice of a 3D sourcefree wind field. This is a common case in the atmosphere, because the 3D wind field is indeed source-free. However, because the flow leaves and enters the slice, the assumption of the absence of sources is no longer valid. Indeed, it is easy to see that the source-free condition for a $3 \mathrm{D}$ wind field, $\boldsymbol{\nabla} \cdot \mathbf{v}=0$, generally does not extend to any of its $2 \mathrm{D}$ slices. For example, in the $x-y$ plane, we have

$$
\boldsymbol{\nabla} \cdot \mathbf{v}_{x y}=\frac{\partial v_{x}}{\partial x}+\frac{\partial v_{y}}{\partial y}=-\frac{\partial v_{z}}{\partial z} \neq 0 .
$$

It should be then concluded that, in this case, both $l_{\Gamma}$ and $t_{\Gamma}$ are needed for the reconstruction and no simplification can be made.

\section{b. Horizontal slice in the stratified atmosphere and source-free wind field}

In the atmosphere, there is a usual stratification caused by gravity and the horizontal component $\mathbf{v}_{x y}$ of wind field is, as a rule, greater than the vertical component $v_{z}$ by factor of 10-100. This gives a possibility to assume sometimes that $v_{z}=0$. More precisely, the vertical component is commonly neglected for temporarily average wind data in micrometeorological studies, if the influence of mesoscale vertical advection (e.g., convection) is neglected. Hence, by inserting this assumption into Eq. (13), the source-free condition in the horizontal plane is now satisfied (i.e., $\boldsymbol{\nabla} \cdot \mathbf{v}_{x y}=0$ ). The wind field $\mathbf{v}_{x y}$ can thus be approximated by the solenoidal field.

We have seen that the longitudinal interaction contains the information on the solenoidal wind field and the problem seems to be solved. However, recall that bounded domains admit harmonic fields that can be seen both as solenoidal or irrotational. For example, Braun and Hauck (1991) proposed to decompose the solenoidal field into the "pure" solenoidal homogeneous component $\mathbf{v}_{S_{0}}$ and the residual harmonic component $\mathbf{v}_{H}$,

$$
\mathbf{v}_{S}=\mathbf{v}_{S_{0}}+\mathbf{v}_{H},
$$

where $\mathbf{v}_{S_{0}}$ is homogeneous in that its normal component is zero on the boundary (completely tangential). In their solution, derived from the original inverse Radon transform, it is shown that, in the case of a circular geometry setup, the obtained result consists of $\mathbf{v}_{S_{0}}$ and only onehalf of $\mathbf{v}_{H}$. The problem that the harmonic field is reconstructed with only one-half of its magnitude can be further treated by reapplying the same inversion to achieve the correct reconstruction. However, the harmonic component will be imagined differently in different geometry setups and the successful reconstruction might not be always possible. Another more general approach was suggested by Norton (1992), where he showed that the measurements of the normal component of $\mathbf{v}_{S}$ taken on the boundary of the region can be used to resolve the ambiguity of the harmonic part.

\section{c. Reconstruction of the source-free wind fields on bounded domain}

Here, we want to show that no additional measurements are required to determine the source-free wind field on the bounded domain. Toward this end, it suffices to prove that the time of flight (in terms of the line integrals) uniquely represents the field. This result is stated in the following theorem.

\section{1) THEOREM 1}

The source-free vector field $\mathbf{v}$ in a bounded simply connected domain $D$ is uniquely determined from the longitudinal interaction through $D$.

\section{2) PROOF 1}

Assume that there exist two different source-free vector fields $\mathbf{v}$ and $\mathbf{u}$ with the same line integrals through $D$ :

$$
\int_{\Gamma} \mathbf{v} \cdot d \mathbf{s}=\int_{\Gamma} \mathbf{u} \cdot d \mathbf{s}, \text { for all } \Gamma \text { in } D .
$$

Applying the 2D version of Stokes' theorem on the difference field $(\mathbf{v}-\mathbf{u})$ and taking $\Gamma=\partial \Omega$ for any $\Omega \in D$, we have

$$
\begin{aligned}
\oint_{\partial \Omega}(\mathbf{v}-\mathbf{u}) \cdot d \mathbf{s} & =\iint_{\Omega} \nabla \times(\mathbf{v}-\mathbf{u}) \cdot \mathbf{e}_{z} d s d l \\
\oint_{\partial \Omega} \mathbf{v} \cdot d \mathbf{s}-\oint_{\partial \Omega} \mathbf{u} \cdot d \mathbf{s} & =\iint_{\Omega} \nabla \times(\mathbf{v}-\mathbf{u}) \cdot \mathbf{e}_{z} d s d l .
\end{aligned}
$$

The left-hand side of Eq. (15) is identically equal to zero by the hypothesis in Eq. (14), because the corresponding line integrals along $\Gamma_{1}-\Gamma_{4}$ (see Fig. 4) are equal. Taking the derivative over $l$ (note that $\Omega$ can change with $l$; e.g., by fixing $\Gamma_{3}$ and moving $\Gamma_{1}(l)$ in the vertical direction), we get 


$$
0=\int_{\Gamma_{1}} \nabla \times(\mathbf{v}-\mathbf{u}) \cdot \mathbf{e}_{z} d s
$$

In the previous equation, $\boldsymbol{\nabla} \times(\mathbf{v}-\mathbf{u}) \cdot \mathbf{e}_{z}$ is a scalar value and does not depend on the mutual orientation of $\Gamma$ and $\mathbf{v}$ - u. Therefore, Eq. (16) coincides with the Radon transform of this value; because it is always zero, we can conclude that the value itself is identical to zero almost everywhere:

$$
\nabla \times(\mathbf{v}-\mathbf{u})=0
$$

Hence,

$$
\mathbf{v}-\mathbf{u}=\nabla \varphi
$$

almost everywhere for some potential function $\varphi$ defined on $D$. Then, it also holds that

$$
0=\int_{\Gamma}(\mathbf{v}-\mathbf{u}) \cdot d \mathbf{s}=\int_{\Gamma} \nabla \varphi \cdot d \mathbf{s}=\varphi\left(\mathbf{x}_{R}\right)-\varphi\left(\mathbf{x}_{T}\right) .
$$

Here, $\Gamma$ is arbitrary, which leads to the conclusion that

$$
\varphi=\text { const. on } \partial D \text {, }
$$

Also, the field $\nabla \varphi$ is by construction irrotational; because it represents the difference of the two solenoidal fields, it is also solenoidal. These two conditions are satisfied only when the field is a harmonic field. The harmonic fields on $D$ satisfy the solutions of the Laplace equation, and they are uniquely determined by its boundary values. Therefore,

$$
\begin{aligned}
\varphi & =\text { const. on } D, \text { and then } \\
\nabla \varphi & =0 \text { on } D,
\end{aligned}
$$

which proves that $\mathbf{v}=\mathbf{u}$, and that the source-free field $\mathbf{v}$ is uniquely determined from its line integrals. QED

The above theorem demonstrates that the line integrals contain sufficient information for the reconstruction of source-free vector fields. Although we show that the transform is injective and therefore the inverse transform exists, we do not provide an explicit inversion formula. Instead, we are going to use algebraic reconstruction methods to reconstruct the unknown field. These methods are discussed in section 4 .

\section{d. Reconstruction of the irrotational wind fields on bounded domains}

It is possible to draw an analogy between the previous case and the case of irrotational wind fields.

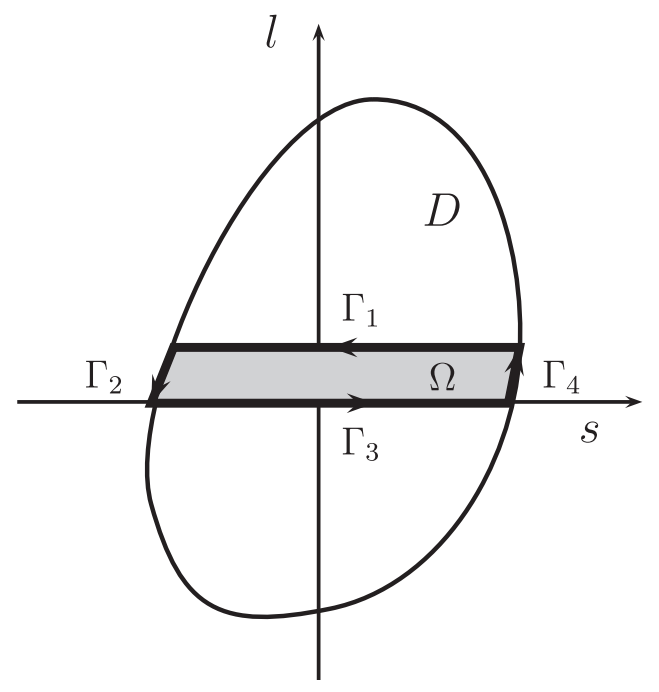

FIG. 4. Figure used in theorem 1 to show that a source-free vector field in a bounded domain $D$ is uniquely determined from its line integrals taken over $D$. The surface $\Omega$ is enclosed by the trajectories $\Gamma_{1}-\Gamma_{4}$.

\section{1) Theorem 2}

The curl-free vector field $\mathbf{v}$ in a bounded simply connected domain $D$ is uniquely determined from the transversal interaction $t_{\Gamma}$ through $D$.

\section{2) Proof 2}

Assume that there exist two different curl-free vector fields $\mathbf{v}$ and $\mathbf{u}$ with the same $t_{\Gamma}$ integrals through $D$,

$$
\int_{\Gamma} \mathbf{v} \cdot d \mathbf{s}_{\perp}=\int_{\Gamma} \mathbf{u} \cdot d \mathbf{s}_{\perp} \quad \text { for all } \Gamma \text { in } D .
$$

Because the fields are irrotational, we have

$$
\begin{aligned}
& \mathbf{v}=\boldsymbol{\nabla} \phi_{1}, \quad \text { and } \\
& \mathbf{u}=\boldsymbol{\nabla} \phi_{2},
\end{aligned}
$$

and the transversal interaction can be rewritten as

$$
\begin{aligned}
& \int_{\Gamma} \mathbf{v} \cdot d \mathbf{s}_{\perp}=\int_{\Gamma} \boldsymbol{\nabla} \times \phi_{1} \mathbf{e}_{z} \cdot d \mathbf{s}=\int_{\Gamma} \mathbf{v}^{\prime} \cdot d \mathbf{s} \text { and } \\
& \int_{\Gamma} \mathbf{u} \cdot d \mathbf{s}_{\perp}=\int_{\Gamma} \boldsymbol{\nabla} \times \phi_{2} \mathbf{e}_{z} \cdot d \mathbf{s}=\int_{\Gamma} \mathbf{u}^{\prime} \cdot d \mathbf{s} .
\end{aligned}
$$

It then also holds that

$$
\int_{\Gamma} \mathbf{v}^{\prime} \cdot d \mathbf{s}=\int_{\Gamma} \mathbf{u}^{\prime} \cdot d \mathbf{s} \quad \text { for all } \Gamma \text { in } D
$$

Applying the results from theorem 1, we have $\mathbf{v}^{\prime}=\mathbf{u}^{\prime}$ and 


$$
\nabla \times \phi_{1} \mathbf{e}_{z}=\nabla \times \phi_{2} \mathbf{e}_{z} .
$$

From the previous equation it also holds that

$$
\nabla \phi_{1}=\nabla \phi_{2} \Rightarrow \mathbf{v}=\mathbf{u}
$$

which proves the claimed result. QED

Theorem 2 proves that the transversal interaction uniquely determines an irrotational wind field on a bounded region. This can be useful in case we are specifically interested in the irrotational wind component.

\section{Reconstruction algorithms}

So far, we have used continuous transforms (e.g., the Radon transform) on which we could choose to apply continuous inversions (e.g., the inverse Radon transform, Fourier slice theorem, and back projection). However, the Radon transform requires the knowledge of the line integrals of the unknown function for all possible lines and all directions. Even its discrete version requires a large number of projections with a special geometric setup (e.g., the projections have to be uniformly distributed over $180^{\circ}$ or $360^{\circ}$ ) to produce results with a reasonable accuracy. An entirely different approach for tomographic reconstruction consists of first assuming that the measured field can be represented by an array of $\mathcal{M}$ parameters $m_{1}, m_{2}, \ldots, m_{M}$ and then setting up a linear set of equations for the unknowns in terms of $\mathcal{D}$ (i.e., the measured data $d_{1}, d_{2}, \ldots$, $\left.d_{N}\right)$. This allows the forward problem in Eq. (1) to be written in the following matrix form:

$$
\mathbf{d}=\mathbf{G m},
$$

where $\mathbf{d}$ and $\mathbf{m}$ are column vectors whose elements are $d_{i}-d_{0 i}$ and $m_{i}-m_{0 i}$, with $i$ being the index of the transmitter-receiver path, and $\mathbf{G}$ is an $N \times M$ matrix whose elements are

$$
G_{i j}=\frac{\partial d_{i}}{\partial m_{j}}
$$

Actually, Eq. (17) is a discretized version of Eqs. (12a) and (12b).

\section{a. Linear versus nonlinear tomography algorithm}

From Eq. (17), we can define two problems. The first one is linear and consists of recovering $\mathbf{m}$, given $\mathbf{G}$ and $\mathbf{d}$. The assumption here is that the ray paths are known a priori. Typically, they are assumed to be straight lines. The second one is nonlinear and consists of recovering
TABLE 1. Nonlinear inverse tomography algorithm.

Algorithm 1: Nonlinear tomography

Step 1: Set the iteration $i=0$ and $\mathbf{m}^{i}$ as an initial model (a constant or the previously best-known model).

Step 2: Compute the trajectories $\hat{\Gamma}$, matrix $\hat{\mathbf{G}}$, and the measurements $\hat{\mathbf{d}}$ for the current model $\mathbf{m}^{i}$.

Step 3: Set $\Delta \mathbf{d}=\mathbf{d}-\hat{\mathbf{d}}$. If $\Delta \mathbf{d}$ is sufficiently small, stop.

Step 4: Find the corrections $\Delta \mathbf{m}$ as the solution of the linear system of equations:*

$$
\hat{\mathbf{G}} \Delta \mathbf{m}=\Delta \mathbf{d} .
$$

Step 5: Update the current version of the model as $\mathbf{m}^{i+1}=\mathbf{m}^{i}+\Delta \mathbf{m}$.

Step 6: Set $i=i+1$ and go to step 2 .

* It is also possible to solve the system $\hat{\mathbf{G m}}^{i+1}=\mathbf{d}$

$\mathbf{m}$ and incidentally $\mathbf{G}$, given $\mathbf{d}$. In this case, the ray paths are unknown and the dependence between the paths and the unknown fields is acknowledged. In nonlinear tomography, an iterative algorithm is needed to find the solution. The iterations alternate between 1) computing an estimate of $\mathbf{m}$ (i.e., $\hat{\mathbf{m}}$ ) and 2) computing the trajectories $\hat{\boldsymbol{\Gamma}}$ that are then used to estimate $\hat{\mathbf{G}}$ and $\hat{\mathbf{d}}$. The next estimate $\hat{\mathbf{d}}$ is then found as a solution of the linear system of equation

$$
\begin{aligned}
& \mathbf{G m}-\hat{\mathbf{G}} \hat{\mathbf{m}}=\mathbf{d}-\hat{\mathbf{d}}, \quad \text { assuming that } \\
& \mathbf{G} \mathbf{m}-\hat{\mathbf{G}} \hat{\mathbf{m}} \simeq \hat{\mathbf{G}}(\mathbf{m}-\hat{\mathbf{m}}) .
\end{aligned}
$$

The basic structure of such an algorithm is given in Table 1 (Sbaiz and Vetterli 2003).

Analyzing the algorithm, we see that there are only two significant calculations contained in it. First, the second step is just the solution of the forward problem and it should not introduce any instability, because it can be performed as accurately as the computing power permits. The fourth step, on the other hand, is crucial for the stability of the algorithm and it is actually the main step both in the linear and nonlinear tomography. The desired solution to the inverse problem will not, in general, be the simple matrix inversion because (i) not all of the data are linearly independent and/or (ii) not all of the models are linearly independent. The latter indicates that there are multiple solutions to the same dataset and the results of the theoretical analysis carried in the previous section should be used to choose the proper datasets and the proper model to avoid this case. Additionally, the matrix $\mathbf{G}$ might be poorly conditioned and the noisy data may prevent finding the exact solution to the system in Eq. (17). We now give a brief overview of available methods. A more thorough 
treatment of inverse scheme can be found in Jovanović (2008).

\section{b. Inverse methods overview}

A common idea behind most of the inversion methods is to minimize the error $\mathbf{e}=\mathbf{d}-\mathbf{G m}$ in some sense.

\section{1) Group 1}

The $l_{2}$-norm minimization is achieved, for example, directly by means of the Moore-Penrose pseudoinverse (Golub and Van Loan 1996), or iteratively, using gradient methods (Moon and Stirling 2000). When the matrix $\mathbf{G}$ is large, the direct inversion is practically limited by computational complexity and memory constraints, and methods referred to as row action or algebraic reconstruction technique (ART; Kak and Slaney 2001) are more attractive. The main idea is that the solution is updated by successively processing each equation separately. Improvements that lead to better convergence are suggested in the simultaneous iterative reconstruction technique (SIRT; Kak and Slaney 2001).

\section{2) Group 2}

Another group of methods tries also to minimize the squared error but in the statistical sense (on average). This results in the stochastic methods based on Wiener or Kalman filtering. In these methods, a priori knowledge about the correlation structure of the solution and the noise is needed. The methods can be extended to include the correlation over space and time (Vecherin et al. 2007).

\section{3) GRoup 3}

Depending on the specific example, the notion of the a priori knowledge can be extended to any other useful information that is available about the field (e.g., the temperature is localized, the wind is a "smooth" function, etc.). In many cases, the information cannot be incorporated into the covariance matrix and other deterministic methods like parametric estimation (Jovanovic et al. $2007 a, b)$ need to be applied. For example, it may be known that in a certain transform domain the model parameters $\mathbf{m}$ have a sparse representation (i.e., $\mathbf{T m}=$ $\mathbf{m}_{s}$, where $\mathbf{m}_{s}$ is a sparse vector). In that case, the new solution $\mathbf{m}_{s}$ to the system

$$
\mathbf{G T}^{\dagger} \mathbf{T} \mathbf{m}=\mathbf{G T}^{\dagger} \mathbf{m}_{s}=\mathbf{d}
$$

is searched as a minimum $l_{0}$-norm solution and it can be achieved by different algorithms as linear programming or convex projections. The concept is known as compressed sensing (Donoho 2006) and has been shown to be very useful for tomographic sampling in general (Jovanović 2008).

In the proposed algorithm, we use a method from the first group, because at this point no a priori information is assumed. The main goal is to show that the approximation steps applied in deriving the longitudinal and the transversal components are valid and allow reconstructing of the temperature and wind fields. Therefore, no regularization is applied and only the pure resolving power of the method is considered.

As the inversion step, we use the conjugate-gradient method with only few iterations (internal iteration of the inversion method). The choice of only a few iterations is motivated by the fact that our goal is not really to solve Eq. (25) in Table 1 but to converge to the solution of the nonlinear problem $\mathbf{G}(\mathbf{m}) \mathbf{m}=\mathbf{d}$. Although the convergence is never insured, it is always "safer" to make a smaller update $\Delta \mathbf{m}$ assuming that the direction of the global minimum is close to the direction of the minimum in Eq. (25).

\section{c. Reconstruction of temperature and full wind field}

To solve the problem, the unknown fields have to be discretized. We define a grid encompassing the tomographic region and assume that the field can be represented by values assigned at the nodes. Some interpolation scheme is then used to find values between the nodes. The limiting case of having infinitely many grid points is equivalent to the continuous case assuming that the underlying field is smooth.

In our algorithms, we use a model in which every point of an unknown parameter $m$ is approximated by a linear combination of the nodal values

$$
m(\mathbf{x})=\sum_{k=1}^{N} m_{k} \alpha_{k}(\mathbf{x}),
$$

where $m_{k}$ is the corresponding value at the node $k$ and $\alpha_{k}(\mathbf{x})$ is an interpolating function. In general, the points between the nodes are interpolated using polynomials of a certain degree. For example, one can cover the domain with a tiling of triangles, in which case $N=3$, and the function $\alpha_{k}(\mathbf{x})$ can be the twodimensional polynomial of order 1 (i.e., the components are approximated by a plane for every triangle). This is a standard interpolation in finite element methods (Brenner and Scott 2002) called linear Lagrange triangle interpolation. The space generated by the nodal variables is the space of two-dimensional 
continuous piecewise linear functions. This space is appropriate if the wind field is represented by its $v_{x}$ and $v_{y}$ components, because the continuity of the field is ensured. If the trajectories are known, Eq. (18) al- lows us to write the set of interactions in Eqs. (12a) and $(12 b)$ as a linear combination of the unknown components $v_{x}, v_{y}$, and $\Delta c$. For the longitudinal component, we have

$$
\begin{aligned}
l_{\Gamma} & =\sum_{j=1}^{J} \int_{\Gamma_{j}}(\Delta c \mathbf{n}+\mathbf{v}) \cdot d \mathbf{s}=\sum_{j=1}^{J} \int_{\Gamma_{j}} \sum_{k=1}^{3} \alpha_{k}(s)\left(\Delta c_{k} \mathbf{n}+\mathbf{v}_{k}\right) \cdot d \mathbf{s} \\
& =\sum_{j=1}^{J} \sum_{k=1}^{3} \underbrace{\left(\int_{\Gamma_{j}} \alpha_{k}(s) \mathbf{n} \cdot d \mathbf{s}\right)}_{a_{k}} \Delta c_{k}+\sum_{j=1}^{J} \sum_{k=1}^{3} \underbrace{\left(\int_{\Gamma_{j}} \alpha_{k}(s) s_{x} d s\right)}_{b_{k}} v_{x, k}+\sum_{j=1}^{J} \sum_{k=1}^{3} \underbrace{\left(\int_{\Gamma_{j}} \alpha_{k}(s) s_{y} d s\right)}_{c_{k}} v_{y, k},
\end{aligned}
$$

where the index $j$ corresponds to the index of the current triangle and $\Gamma_{j}=\Gamma \cap$ triangle $_{j}$. The index $k$ denotes the vortex index within the current triangle. A similar set of equations can be developed for the transversal components by exchanging vector $\mathbf{s}$ with vector $\mathbf{s}_{\perp}$. In matrix notation, we can write

$$
\left(\begin{array}{l}
\mathbf{G}_{l} \\
\mathbf{G}_{t}
\end{array}\right) \cdot \mathbf{m}=\left(\begin{array}{l}
\mathbf{l} \\
\mathbf{t}
\end{array}\right),
$$

where $\mathbf{m}^{\mathrm{T}}=\left(\mathbf{v}_{x}^{\mathrm{T}} \mathbf{v}_{y}{ }^{\mathrm{T}} \Delta \mathbf{c}^{\mathrm{T}}\right)$ and $\mathbf{l}$ and $\mathbf{t}$ are the vectors with the longitudinal and transversal component, respectively. The matrices $\mathbf{G}_{l}$ and $\mathbf{G}_{t}$ describe the linear relationship between the measurements and the unknowns, as given in Eq. (19). Equation (20) can be then solved by applying algorithm 1 , which is given in section $4 \mathrm{a}$.

\section{d. Reconstruction of temperature and source-free wind field}

In section $4 \mathrm{~b}$, it is shown that the longitudinal interaction allows the recovery of the temperature and 2D source-free wind field. Therefore, the system in Eq. (20) can be reduced to

$$
\mathbf{G}_{l} \cdot \mathbf{m}=\mathbf{l}
$$

In the previous case, we chose to represent the wind field as $\mathbf{v}=\left[v_{x}, v_{y}\right]$. However, this representation does not reflect the fact that the unknown field has only a solenoidal component. Without this constraint, the system in Eq. (21) is not invertible and admits an infinite number of solutions. On the contrary, the representation with the potentials $\psi$, more precisely with the derivatives of $\psi$ (i.e., $\mathbf{v}=\boldsymbol{\nabla} \times \psi \mathbf{e}_{z}=[\partial \psi / \partial y,-\partial \psi / \partial x]$ ), automatically imposes the source-free condition and discards all invisible winds. To ensure the continuity of the wind field, $\psi$ and its derivatives need to be continuous. Therefore, the linear Lagrange triangles are no longer appropriate and we choose another finite element, namely the reduced Hseih-Clogh-Tocher (HCT) triangles (Braess 2001). The HCT interpolates the third-order polynomial function that is continuous together with its first-order derivatives. In the HCT representation, the region of interest is covered with the triangular tiles and the points inside the triangles are parameterized using the value of the three vortex nodes $m_{k}$, and six directional first-order derivatives $\partial m / \partial x, \partial m / \partial y$ (two per node):

$$
m(\mathbf{x})=\sum_{k=1}^{9} m_{k} \alpha_{k}(\mathbf{x}) .
$$

This more complex tessellation adds complexity to the algorithm because now every triangle is determined by nine parameters, but the reconstructed wind is a secondorder polynomial function, whereas the reconstructed temperature is a third-order polynomial. The coefficients in $\mathbf{G}_{l}$ have to be computed accordingly to the new representation but similarly as before:

$$
\begin{aligned}
l_{\Gamma} & =\int_{\Gamma}\left(\Delta c \mathbf{n}+\nabla \times \psi \mathbf{e}_{z}\right) \cdot d \mathbf{s}=\sum_{j=1}^{J} \int_{\Gamma_{j}}\left(\Delta c \mathbf{n}+\nabla \times \psi \mathbf{e}_{z}\right) \cdot d \mathbf{s} \\
& =\sum_{j=1}^{J} \int_{\Gamma_{j}}\left(\sum_{k=1}^{9} \alpha_{k}(s) \Delta c_{k}\right) \mathbf{n} \cdot \mathbf{s}+\nabla \times\left(\sum_{k=1}^{9} \beta_{k}(s) \psi_{k}\right) \mathbf{e}_{z} \cdot \mathbf{s} d s,
\end{aligned}
$$


where $\psi_{k}$ and $\Delta c_{k}$ are now the unknowns in the HCT model. The previous equation is then incorporated in the global nonlinear algorithm 1 .

\section{Simulation results}

The simulation setup consists of a circular array that is $10 \mathrm{~m}$ in diameter; it is equipped with 20 transmitters and 20 receivers. Every transmitter sends a signal to every receiver, which results in 400 transmission paths. The region of interest is covered with triangular cells of the same size. Ideally, to resolve the sound speed and the wind field at one nodal point the number of transmissions affected by that node needs to be equal or greater than the number of unknowns that characterize the node, and also the total number of transmission paths needs to be equal or greater than the total number of unknowns. However, if no a priori knowledge is considered, the system of equations to be solved is usually poorly conditioned. It can be stabilized by having more measurements than the number of unknowns.

We first simulate the forward problem. Our code implements Eqs. (2) and (3) for the ray tracing in an inhomogeneous moving medium. A fifth-order RungeKutta algorithm is applied to integrate the ray equations forward in time. The shooting method is used to search for the correct ray. The algorithm has an adjustable integration step size and error tolerance at the receiver side. The resulting time-of-flight and angle-of-departure/ arrival measurements are then taken as the measurement set. The solution is computed using the iterative algorithm described in section 4 , with the conjugate-gradient method in the fourth step of Table 1 .

\section{a. Error analysis}

To evaluate the effect of noisy measurements we add some error to both the time-of-flight and angle-ofdeparture/arrival measurements. For a specific signalto-noise ratio (SNR) at the received signal, we can estimate the error in the measurements as follows.

When the acoustic dipoles are used for measuring the angle of arrivals, the error is determined by the error in the time of flight and the error in the distance between the two receivers in the acoustic dipole. Therefore, let us first compute the Cramer-Rao lower bound of the time-of-flight estimates. This bound determines the lower bound for the error standard deviation $\sigma_{\tau}$, and it is actually admitted when the time of flight is estimated from the peak of the cross correlation between the sent and the received signal. In Woodward (1953), it is computed that

$$
\sigma_{\tau}^{2} \geq \frac{1}{8 \pi^{2}} \frac{1}{\operatorname{SNR}} \frac{1}{T_{o} B} \frac{1}{f_{c}^{2}} \frac{1}{\left(1+B^{2} / 12 f_{c}^{2}\right)},
$$
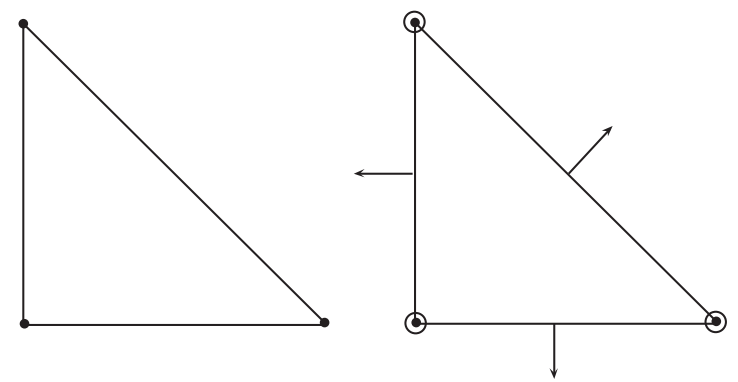

FIG. 5. (left) Lagrange and (right) HCT triangles, where dots denote the values, circles denote the first derivative, and arrows denote the first directional derivatives.

where $T_{o}$ is the observation time, $f_{c}$ the central frequency, and $B$ the signal bandwidth. If we change the parameters in Eq. (22) with the ones that we use in our experiments $\left(T_{o}=2 \mathrm{~ms}, f_{c}=40 \mathrm{kHz}\right.$, and $B=2 \mathrm{kHz}$; Jovanović et al. 2006) and for the two cases of SNR, we get ${ }^{2}$

$$
\begin{aligned}
& \mathrm{SNR}=30 \mathrm{~dB} \rightarrow \sigma_{\tau} \simeq 4.4 \times 10^{-8} \mathrm{~s} \quad \text { and } \\
& \mathrm{SNR}=10 \mathrm{~dB} \rightarrow \sigma_{\tau} \simeq 4.4 \times 10^{-7} \mathrm{~s} .
\end{aligned}
$$

We can further use the previous result to compute the error of the angle-of-arrival estimates. In an acoustic dipole, the angle is computed from the time difference

$$
\cos \theta=\frac{\left(\tau_{1}-\tau_{2}\right) c_{0}}{d}
$$

where $d$ is the distance between the two receivers in the dipole. The error standard deviation is then equal to

$$
\sigma_{\cos \theta}=\frac{2 \sigma_{\tau} c_{0}}{d}
$$

Assuming that $d=0.1 \mathrm{~m}$ and $c_{0}=334 \mathrm{~m} \mathrm{~s}^{-1}$, we find

$$
\begin{aligned}
& \mathrm{SNR}=30 \mathrm{~dB} \rightarrow \sigma_{\cos \theta}=2.9 \times 10^{-4} \text { and } \\
& \mathrm{SNR}=10 \mathrm{~dB} \rightarrow \sigma_{\cos \theta}=2.9 \times 10^{-3} .
\end{aligned}
$$

The error in the angle measurements is then

$$
\sigma_{\theta}=\frac{\sigma_{\cos \theta}}{\sin \theta}
$$

The error added to the angles of departure/arrival is then generated as a uniformly distributed white noise with the standard deviation computed in the previous equations.

\footnotetext{
${ }^{2}$ Note that the SNR in Eq. (22) represents the signal energy over the noise energy $\left(\mathrm{S}^{2} / \mathrm{N}^{2}\right)$ and it is not given in decibels, which we have used for later comparison.
} 


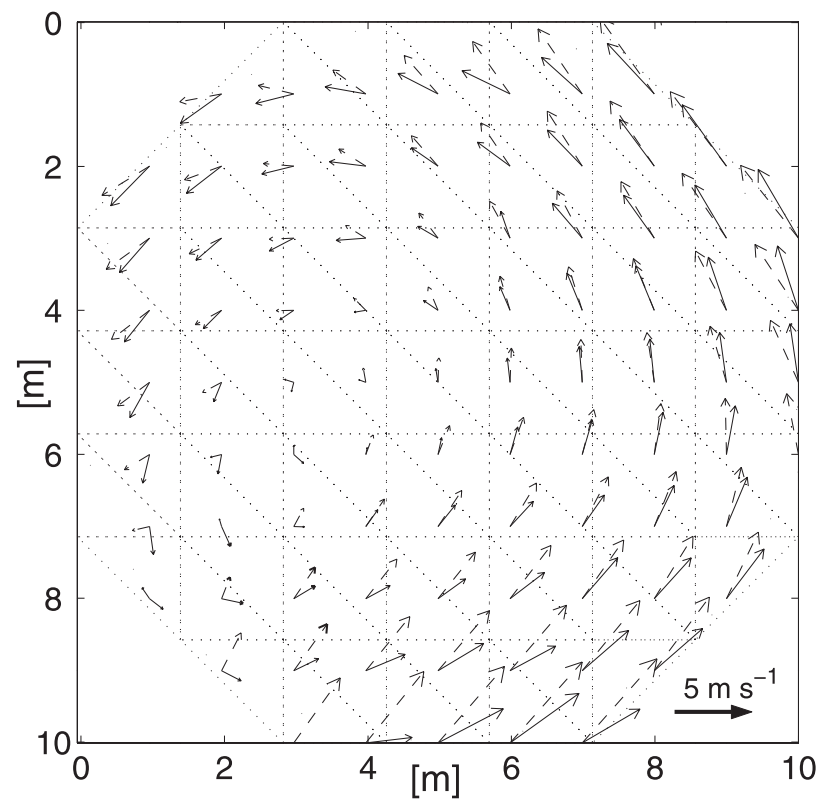

(a)

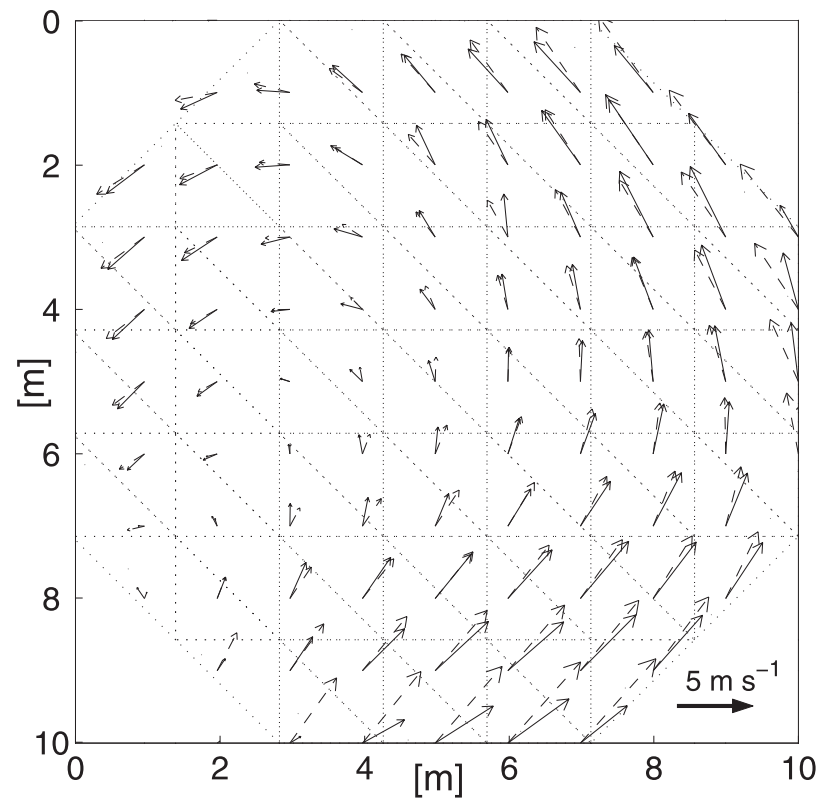

(c)

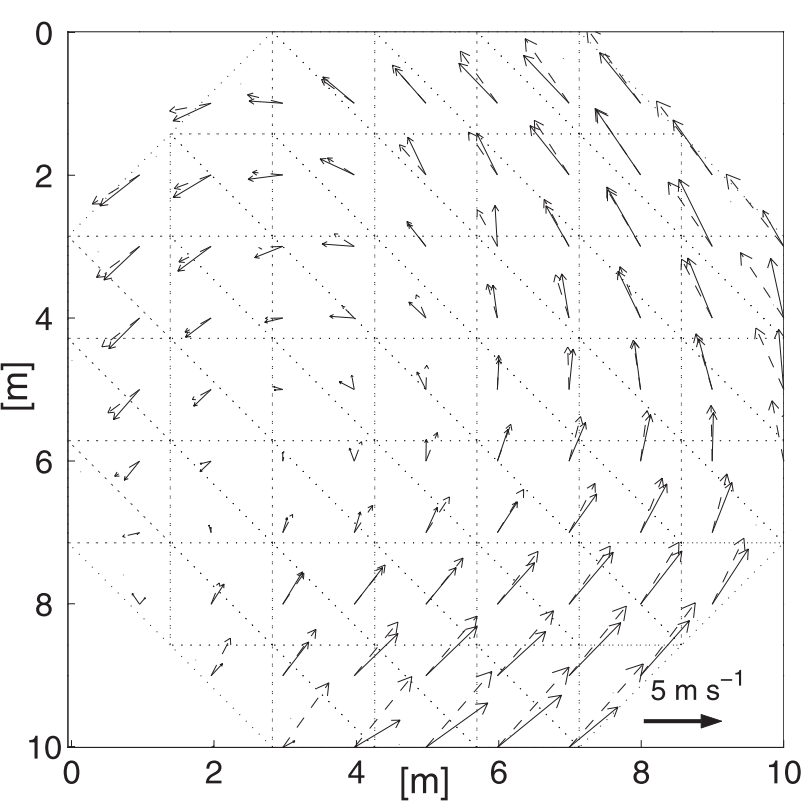

(b)

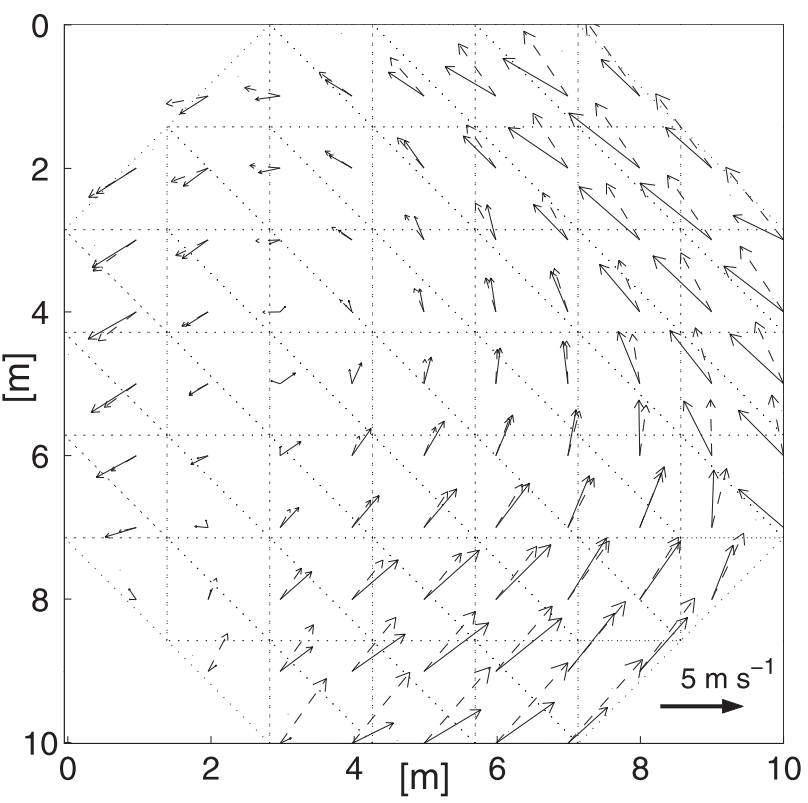

(d)

FIG. 6. Original (dashed) and reconstructed (solid) wind field for (a) the straight-ray model, with rmse ${ }_{v}=1.63 \mathrm{~m} \mathrm{~s}^{-1}$; (b) the bent-ray model, with $\mathrm{rmse}_{v}=1.35 \mathrm{~m} \mathrm{~s}^{-1}$; (c) the bent-ray model with noise, with SNR $=30 \mathrm{~dB}$ and rmse ${ }_{v}=2.28 \mathrm{~m} \mathrm{~s}^{-1}$; and (d) the bent-ray model with noise, with SNR $=20 \mathrm{~dB}$ and rmse $_{v}=2.46 \mathrm{~m} \mathrm{~s}^{-1}$. 


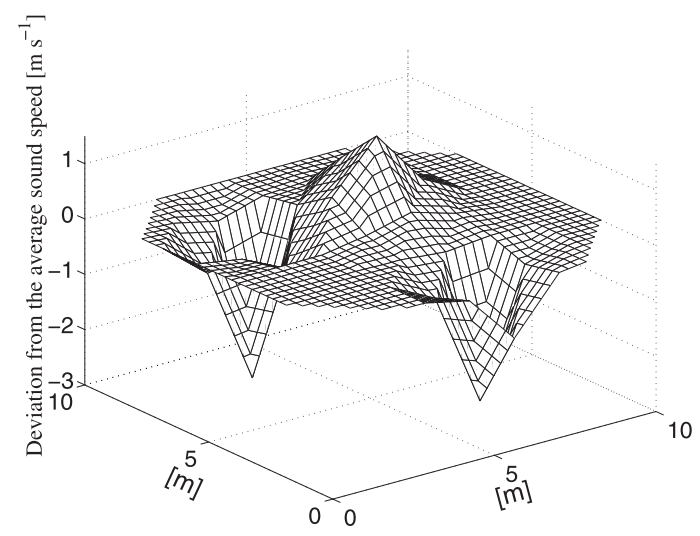

(a)

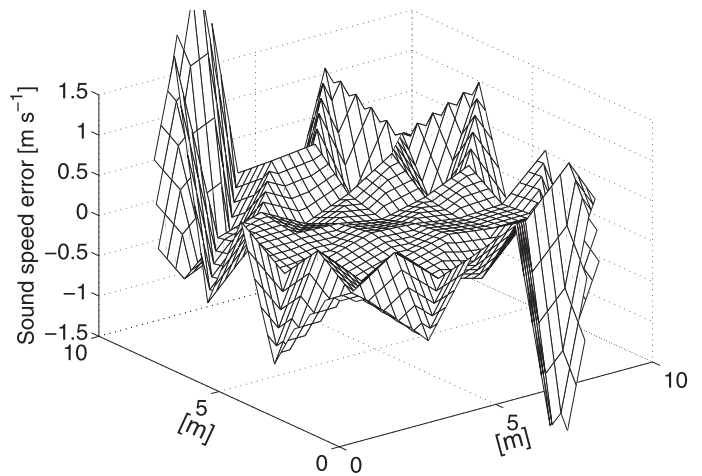

(b)

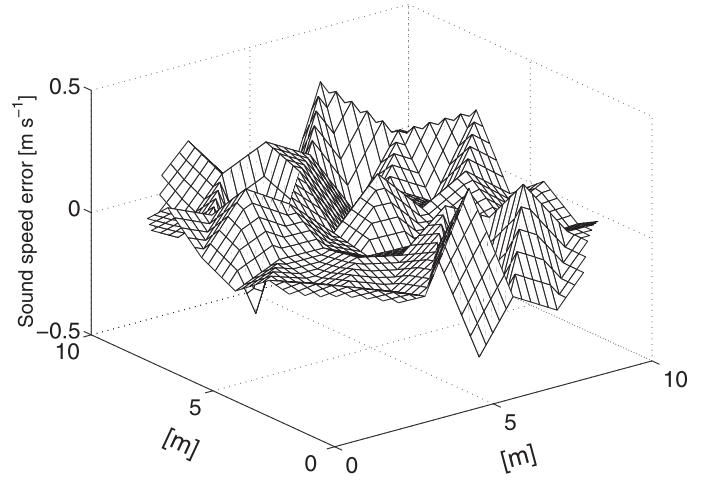

(d)

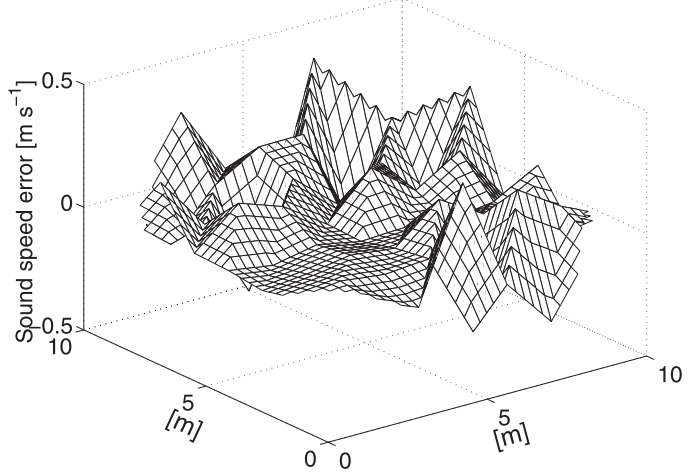

(c)

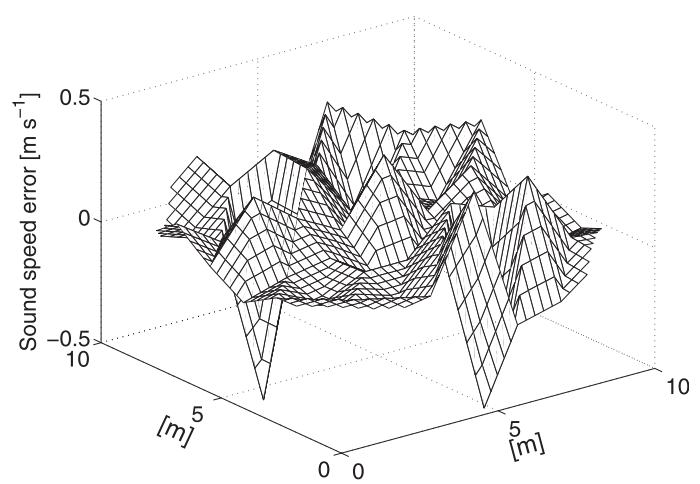

(e)

FIG. 7. Deviation from the average sound speed for (a) true sound speed deviation and reconstruction error using (b) the straight-ray model, with $\mathrm{rmse}_{c}=1.95 \mathrm{~m} \mathrm{~s}^{-1}$; (c) the bent-ray model, with rmse ${ }_{c}=0.81 \mathrm{~m} \mathrm{~s}^{-1}$; (d) the bent-ray model with noise, with SNR $=30 \mathrm{~dB}$ and $\mathrm{rmse}_{c}=2.10 \mathrm{~m} \mathrm{~s}^{-1}$; and (e) the bent-ray model with noise, with $\mathrm{SNR}=20 \mathrm{~dB}$ and $\mathrm{rmse}_{c}=2.02 \mathrm{~m} \mathrm{~s}^{-1}$. 
TABLE 2. Comparative error for temperature and full wind reconstruction.

\begin{tabular}{|c|c|c|c|c|}
\hline & \multicolumn{2}{|c|}{ Straight ray } & \multicolumn{2}{|c|}{ Bent ray } \\
\hline & $\mathrm{rmse}_{v}\left(\mathrm{~m} \mathrm{~s}^{-1}\right)$ & $\operatorname{rmse}_{c}\left(\mathrm{~m} \mathrm{~s}^{-1}\right)$ & $\mathrm{rmse}_{v}\left(\mathrm{~m} \mathrm{~s}^{-1}\right)$ & $\mathrm{rmse}_{c}\left(\mathrm{~m} \mathrm{~s}^{-1}\right)$ \\
\hline No noise & 1.63 & 1.95 & 1.35 & 0.81 \\
\hline $\mathrm{SNR}=30 \mathrm{~dB}$ & 3.51 & 2.10 & 2.28 & 2.34 \\
\hline $\mathrm{SNR}=20 \mathrm{~dB}$ & 3.9 & 2.02 & 2.46 & 2.55 \\
\hline $\mathrm{SNR}=15 \mathrm{~dB}$ & 3.67 & 2.07 & 2.66 & 2.59 \\
\hline
\end{tabular}

\section{b. Results}

\section{1) NuMERICAL EXAMPLE 1 (TEMPERATURE AND FULL WIND)}

In this example, we want to reconstruct a general full wind and temperature field using the time-of-flight and the angle-of-departure/arrival measurements. The region of interest is covered with the Lagrangian triangles (see Fig. 5). In every iteration, we aim at solving the linearized system in Eq. (20). Figure 6a shows the original wind field and the reconstructed wind field, which is obtained after the first iteration and using the straight-ray model. The arrows represent the amplitude and the direction of the wind. The maximal wind speed is $5 \mathrm{~m} \mathrm{~s}^{-1}$. The root mean squared error (rmse) of the wind reconstruction in this example is rmse $_{v}=$ $1.63 \mathrm{~m} \mathrm{~s}^{-1}$. Figure $6 \mathrm{~b}$ shows the original and reconstructed wind fields when the bent-ray model is used. The rmse lowers to $\mathrm{rmse}_{v}=1.35 \mathrm{~m} \mathrm{~s}^{-1}$. This shows that the bent-ray model and the nonlinear iterative algorithm outperform the straight-ray model. Further, the noise is added to the angle-of-arrival measurements. In Fig. 6c, the assumed SNR is $30 \mathrm{~dB}$ (corresponds to $\left.\sigma_{\cos \theta}=2.9 \times 10^{-4}\right)$, which results in $\mathrm{rmse}_{v}=2.28 \mathrm{~m} \mathrm{~s}^{-1}$. Figure $6 \mathrm{~d}$ shows the original and the reconstructed wind field when SNR $=20 \mathrm{~dB}$ (corresponds to $\sigma_{\cos \theta}=9.2 \times$ $\left.10^{-4}\right)$, which induces $\mathrm{rmse}_{v}=2.46 \mathrm{~m} \mathrm{~s}^{-1}$.

The original sound speed variation is shown in Fig. 7a. The reconstruction errors for the straight- and bent-ray models are represented in Fig. $7 \mathrm{~b}\left(\mathrm{rmse}_{c}=1.63 \mathrm{~m} \mathrm{~s}^{-1}\right)$ and Fig. 7c $\left(\mathrm{rmse}_{c}=0.81 \mathrm{~m} \mathrm{~s}^{-1}\right)$, respectively. The reconstruction errors for the noisy received signals, with $\mathrm{SNR}=30 \mathrm{~dB}$ and SNR $=20 \mathrm{~dB}$, are represented in Figs. $7 d$,e, respectively. Such as for the wind field, the reconstruction with the bent-ray model outperforms the straight-ray model reconstruction. A comparative error overview is given in Table 2, and it additionally includes the rmse for the SNR $=15 \mathrm{~dB}$ and the straight-ray model with noise.

\section{2) NUMERICAL EXAMPLE 2 (TEMPERATURE AND SOURCE-FREE WIND)}

In the second example, it is assumed that the 2D slice of a wind field is source-free. The temperature and the wind field are reconstructed from the time-of-flight measurements. The measurement setup is identical to the previous one, except that, in this case, the region of interest is covered by the HCT triangles (see Fig. 5). We assume that the SNR is 30 and $10 \mathrm{~dB}$ and corresponds to $\sigma_{\tau}=4.4 \times 10^{-8}$ and $\sigma_{\tau}=4.4 \times 10^{-7}$, respectively. The original wind and the reconstructed wind fields with noisy data are shown in Fig. 8. For SNR $=30 \mathrm{~dB}$, the difference between the original and reconstructed wind fields is not noticeable. Only a slight difference can be seen for SNR $=10 \mathrm{~dB}$. This proves that the reconstructions are robust to the error in the time-of-flight measurements. The original sound speed variation and the corresponding reconstructions are shown in Figs. 9. As for the wind, the results show to be robust to the error in the time-of-flight measurements.

For both examples, the error inside the region of interest is usually smaller than the error on the border. This is because the points inside the region are common to many trajectories; hence, more information about these points is available. Therefore, we can say that the points inside are better conditioned (in terms of the condition number) than the points on the border. Consequently, because the error in the measurements translates to the error in the reconstruction proportionally to the inverse of the condition number, the error on the border is always higher than the error inside the region of interest.

The idea of performing only a few (one or two inner) iterations in the conjugate-gradient method showed to be successful. Numerical simulations confirmed that the iterative algorithm achieves better reconstruction than the one-step inversion. For both examples, the iterative algorithm stops after at most 5 iterations. The stopping conditions are defined based on 1 ) the error between the measurements and the forward prediction of the current system, 2) the number of iterations, and 3) the variation in the solution over the past iterations.

\section{Conclusions}

The goal of this paper was to cover important points related to the use of $2 \mathrm{D}$ acoustic tomography for estimating temperature and wind in the atmosphere. It 


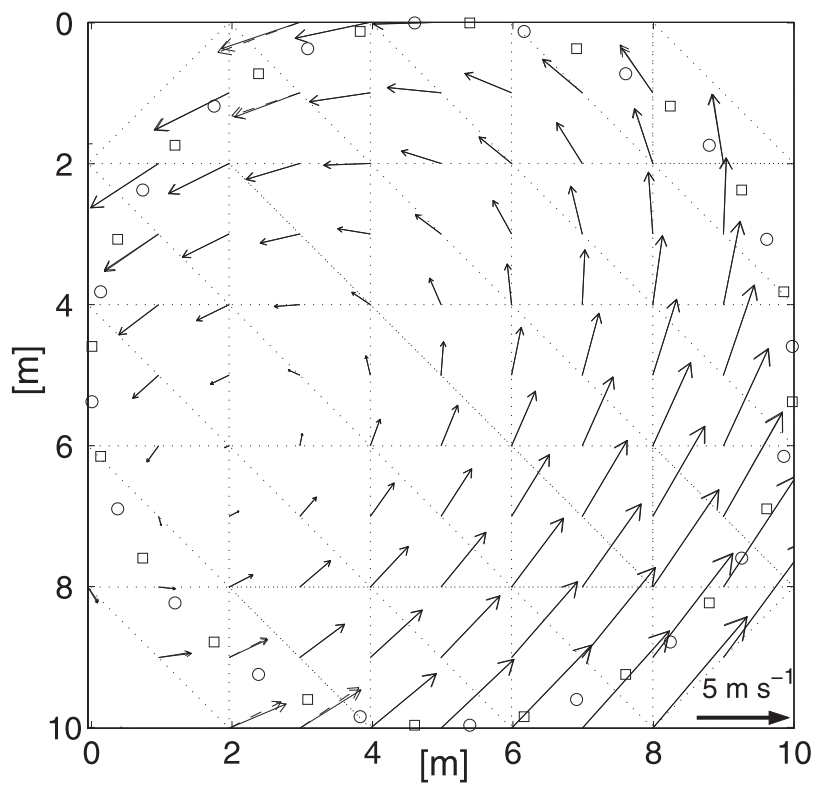

(a)

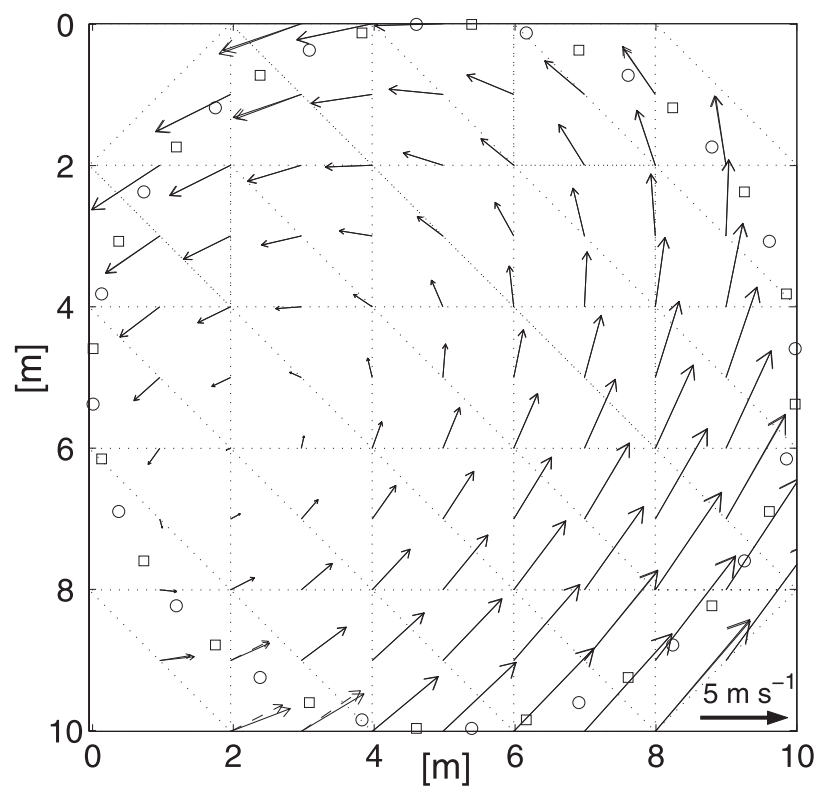

(b)

FIG. 8. Original (dashed) and reconstructed (solid) wind field for (a) the original and reconstructed wind field overlap for SNR $=30 \mathrm{~dB}$ of the received signal and (b) the original and reconstructed wind fields for SNR $=10 \mathrm{~dB}$ of the received signal.

aimed in setting up the inverse problem properly and showing the techniques for obtaining the solution. Particularly, we showed that, when estimating a 2D slice of temperature and wind, the time-of-flight measurements are not sufficient. Only the cases of source-free 2D wind fields (that are not automatically extended from a 3D source-free condition) can be faithfully reconstructed. Otherwise, a new set of measurements related to the angles of departure/arrival of a sound wave is needed. We showed how these measurements can be obtained in practice. When an acoustic tripole is used, the angles of departure/arrival are estimated from the time-of-flight measurements. Hence, in that case, the full reconstruction can be realized using only the time-of-flight measurements.

Numerical simulations confirmed that the approximation steps applied in deriving the longitudinal and transversal interactions lead to a good reconstruction. Also, the bent-ray model outperforms the straight-ray model. An interesting question was to see how the noise in the measurements affects the reconstruction. $\mathrm{Nu}$ merical simulations showed that the algorithms are robust to the error in the time-of-flight measurements (see numerical example 2). The method is less robust to the error in the angle-of-departure/arrival measurements. Simulation results showed that the additional measurements improved the reconstruction when the received signal has the $\mathrm{SNR} \geq 20 \mathrm{~dB}$. For smaller SNR, the improvement of the bent-ray reconstruction is smaller compared to the reconstruction with the straight rays, for which we do not need additional angle-ofdeparture/arrival measurements. However, this observation is dependent on the specific example that we consider, and the choice between the straight-ray (no angle-of-departure/arrival measurements and linear tomography) and bent-ray (additional angle-of-departure/ arrival measurements and nonlinear tomography) methods needs to be taken by considering the following questions: 1) In the given scenario, can we neglect the downdraft and the updraft winds? Equivalently, how strong is the irrotational wind field? 2) What error in the measurements can we expect? Consequently, given this error, is the use of more precise bent-ray algorithms justified? It is worth studying these questions in relation to specific practical situations (e.g., flat terrain versus complex terrain), and we envision these topics in our future research.

We also studied the problem of recovering source-free vector fields. As opposed to the previously suggested solution, we show that the time-of-flight measurements are indeed sufficient for the reconstruction. However, to obtain a correct solution, it is important to include the source-free condition in the reconstruction process. As an example, we referred to the case of estimating a horizontal slice of the wind field in the stratified atmosphere. Numerical simulation showed that the method is robust to the error in the time-of-flight measurements. 


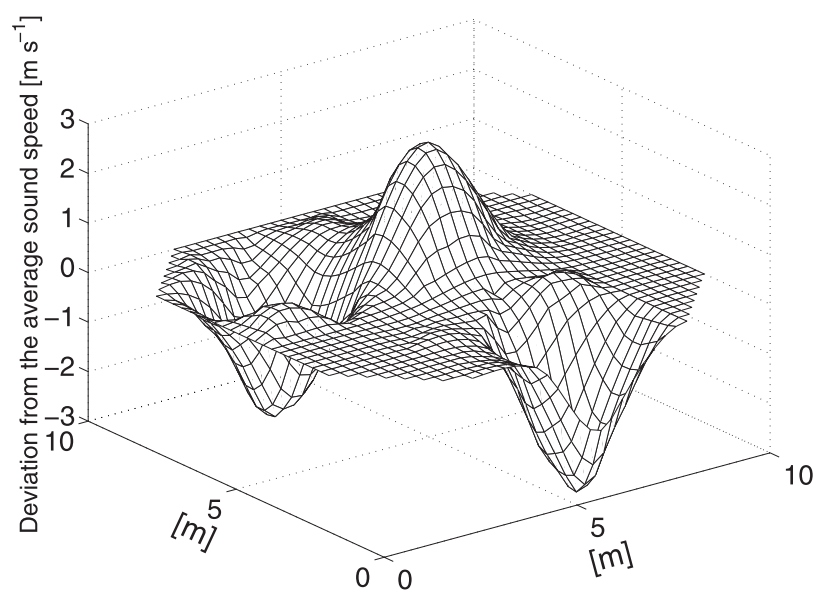

(a)

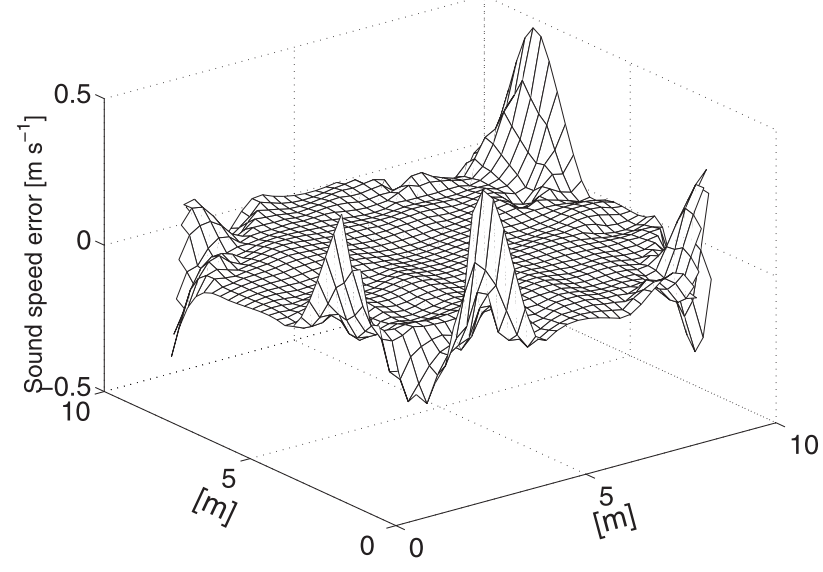

(b)

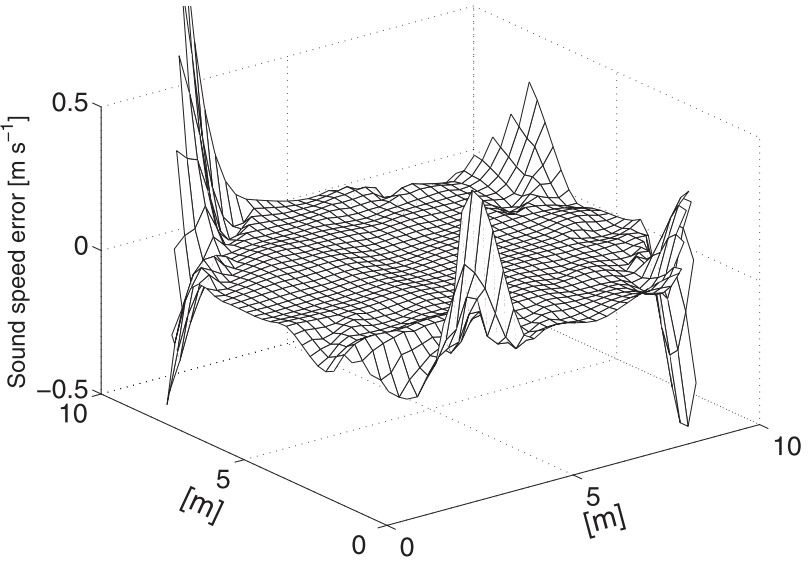

(c)

FIG. 9. Deviation from the average sound speed: (a) original sound speed variation; (b) reconstruction error from the noisy received signal SNR $=30 \mathrm{~dB}$; and $(\mathrm{c})$ reconstruction error from the noisy received signal $\mathrm{SNR}=10 \mathrm{~dB}$.

From a practical point of view, acoustic tomography showed to be a powerful method for studying smallscale temperature and wind distributions in the atmosphere with a high spatial resolution. In this context, it can be a very important tool in the study and validation of the theories of turbulence flow. One method for reconstructing turbulent flow based on acoustic tomography is already suggested in Vecherin et al. (2007). Although, the authors ignore the main results of this paper (that the time-of-flight measurements are not sufficient for the full wind reconstruction), the exposed ideas can be useful when applied with appropriate changes. The time resolution in acoustic tomography is high as well, because the information is obtained without interaction with the atmosphere and without inertia. Still, the time and effort to apply the tomographic method outside the laboratory are high. One of the problems is that a precise calibration should take into account all delays in the system. However, the precise calibration can be avoided when only a variation of sound speed is monitored. In that case, the variation is calculated with respect to the measurements taken at some referent time instant.

Acknowledgments. This work was supported by the Swiss National Science Foundation under Grant 205321122240/1.

\section{REFERENCES}

Barth, M., K. Arnold, and A. Raabe, 2006: Flow field detection using acoustic time tomography. Proc. 13th Int. Symp. for the Advancement of Boundary Layer Remote Sensing, GarmischPartenkirchen, Germany, Institute of Physics, 81-82. 
Braess, D., 2001: Finite Elements: Theory, Fast Solvers, and Applications in Solid Mechanics. Cambridge University Press, $352 \mathrm{pp}$.

Braun, H., and A. Hauck, 1991: Tomographic reconstruction of vector fields. IEEE Trans. Signal Process., 39, 464-471.

Brenner, S. C., and L. R. Scott, 2002: The Mathematical Theory of Finite Element Method. Springer-Verlag, 361 pp.

Chunchuzov, I. P., A. I. Otrezov, I. V. Petenko, V. N. Tovchigrechko, A. I. Svertilov, A. L. Fogel, and V. E. Fridman, 1997: Traveltime and duration fluctuation of acoustic pulses in the atmospheric boundary layer. Izv. Atmos. Oceanic Phys., 3, 324-338.

Coppin, P. A., and K. J. Taylor, 1983: A three-component sonic anemometer/thermometer system for general micrometeorological research. Bound.-Layer Meteor., 27, 27-42.

Donoho, D. L., 2006: Compressed sensing. IEEE Trans. Inf. Theory, 52, 1289-1306.

Golub, G., and C. F. Van Loan, 1996: Matrix Computations. Johns Hopkins University Press, 694 pp.

Haacke, E. M., R. W. Brown, M. R. Thompson, and R. Venkatesan, 1999: Magnetic Resonance Imaging: Physical Principles and Sequence Design. Wiley-Liss, 914 pp.

Johnson, S. A., J. F. Greenleaf, C. R. Hansen, W. F. Samayoa, A. L. M. Tanaka, D. A. Christensen, and R. L. Wooley, 1977: Reconstructing three-dimensional fluid velocity vector fields from acoustic transmission measurements. Acoustical Holography, L.W. Kessler, Ed., Vol. 7, Plenum, 307-326.

Jovanović, I., 2008: Inverse problem in acoustic tomography: Theory and applications. Ph.D. thesis, Ecole Polytechnique Fédérale de Lausanne (EPFL), 139 pp.

—, L. Sbaiz, and M. Vetterli, 2006: Acoustic tomography for estimating temperature and wind flow. Proc. 13th Int. Symp. for the Advancement of Boundary Layer Remote Sensing, Garmisch-Partenkirchen, Germany, Institute of Physics, 69-71. , A. Hormati, L. Sbaiz, and M. Vetterli, 2007a: Efficient and stable acoustic tomography using sparse reconstruction methods. Proc. 19th Int. Congress on Acoustics, Madrid, Spain, PACS number 43.30.Pc. [Available online at http://infoscience.epfl.ch/ record/104545/files/.]
_ L. Sbaiz, and M. Vetterli, 2007b: Tomographic approach for parametric estimation of local diffusive sources and application to heat diffusion. Proc. 14th Int. Conf. on Image Processing, San Antonio, TX, IEEE, 153-156.

Kak, A. C., and M. Slaney, 2001: Principles of Computerized Tomographic Imaging. Society of Industrial and Applied Mathematics, $327 \mathrm{pp}$.

Moon, T. K., and W. C. Stirling, 2000: Mathematical Methods and Algorithms for Signal Processing. Prentice Hall, 937 pp.

Munk, W., and C. Wunsch, 1982: Observing the ocean in the 1990s. Philos. Trans. Roy. Soc. London, 307, 439-464.

Norton, S. J., 1992: Unique tomographic reconstruction of vector fields using boundary data. IEEE Trans. Image Process., 1, 406-412.

Ostashev, V. E., 1997: Acoustics in Moving Inhomogeneous Media. E\&FN SPON, 259 pp.

Sbaiz, L., and M. Vetterli, 2003: Acoustic flow tomography. Audiovisual Ecole Polytechnique Fédérale de Lausanne (EPFL) Communications Laboratory (LCAV) Tech. Rep., 16 pp.

Spiesberger, J. L., and K. M. Fristrup, 1990: Passive localization of calling animals and sensing of their acoustic environment using acoustic tomography. Amer. Nat., 135, 107-153.

Stewart, R. R., 1991: Exploration Seismic Tomography: Fundamentals. Course Notes Series, Vol. 4, Society of Exploration Geophysicists, 140 pp.

Vecherin, S. N., V. E. Ostashev, A. Ziemann, D. K. Wilson, K. Arnold, and M. Barth, 2007: Tomographic reconstruction of atmospheric turbulence with the use of time-dependent stochastic inversion. J. Acoust. Soc. Amer., 122, 1416-1425.

Wilson, D. K., and D. W. Thomson, 1994: Acoustic tomographic monitoring of the atmospheric surface layer. J. Atmos. Oceanic Technol., 11, 751-769.

Woodward, P. M., 1953: Probability and Information Theory, with Applications to Radar. Pergamon Press, 128 pp.

Ziemann, A., K. Arnold, and A. Raabe, 1999a: Acoustic tomography in the atmospheric surface layer. Ann. Geophys., 17, 139-148.

,$- \ldots$, and $-1999 \mathrm{~b}$ : Acoustic travel time tomography-A method for remote sensing of the atmospheric surface layer. Meteor. Atmos. Phys., 71, 43-51. 